\title{
THE MORSE LEMMA IN INFINITE DIMENSIONS VIA SINGULARITY THEORY*
}

\author{
MARTIN GOLUBITSKY ${ }^{\dagger}$ AND JERROLD MARSDEN ${ }^{\ddagger}$
}

\begin{abstract}
An infinite dimensional Morse lemma is proved using the deformation lemma from singularity theory. It is shown that the versions of the Morse lemmas due to Palais and Tromba are special cases. An infinite dimensional splitting lemma is proved. The relationship of the work here to other approaches in the literature in discussed.
\end{abstract}

Introduction. This paper shows that when the singularity theory proof of the Morse lemma is extended to infinite dimensions, it gives a result better than the best available. The best available Morse lemma is that of Tromba [1976], [1981] which improves upon the usual Morse-Palais lemma (cf. Palais [1963], [1969]) for the following crucial reason: The Morse-Palais lemma assumes that the second derivative of the function at its critical point is strongly nondegenerate in the sense of defining an isomorphism between the space and its dual. Such a hypothesis is not satisfied in standard elliptic variational problems; however, the hypotheses of Tromba's Morse lemma are normally verified in such problems. Specific examples are presented in Buchner, Marsden and Schecter [1983]; for others see

(a) Tromba [1976], [1981] for geodesics and minimal surfaces;

(b) Choquet-Bruhat and Marsden [1976] and Arms, Marsden and Moncrief [1982] for general relativity;

(c) Ball, Knops and Marsden [1978] and Marsden and Hughes [1983] for elasticity.

In conjunction with the Morse lemma are questions of

1. normal forms for more degenerate singularities and

2. a splitting lemma and reduction to finite dimensional catastrophe theory.

Such questions have been studied by Magnus [1976], [1978], [1979], Arkeryd [1979] and Chillingworth [1980], but under hypotheses similar to those of the Morse-Palais lemma. In view of the difficulties with these hypotheses, it is important to also carry this program out under more applicable hypotheses. Such a setting is provided here. A related setting for a normal form theory in infinite dimensions is presented in Beeson and Tromba [1981]. Their situation is further complicated by the presence of a group action. A closely related setting is given in Dangelmayr [1979] and Magnus [1980].

The plan of the paper is as follows:

1. Theorem $\mathrm{A}$ in $\$ 2$ gives conditions under which two given functions are related by a diffeomorphism in a neighborhood of a singular point.

2. Theorem B in $\S 3$ is the Morse-Tromba lemma and is shown to be a straightforward consequence of Theorem A.

3. Section 4 discusses the splitting lemma and the associated reduction to finite dimensional catastrophe theory.

Finally, we note that the ideas in Theorem A below are useful in the study of vector fields. In particular, the methods can be used to deal with some $C^{\infty}$-flat ambiguities in normal forms of vector fields at a singular point. These topics will be the subject of other publications.

*Received by the editors June 28, 1982, and in revised form October 29, 1982.

${ }^{\dagger}$ Department of Mathematics, University of Houston, Houston, Texas 77004.

${ }^{\ddagger}$ Department of Mathematics, University of California, Berkeley, California 94720. 
1. The singularity theory method. To put the methods in perspective, we shall recall some of the ideas of singularity theory with a view towards the Morse lemma. The basic methods of singularity theory under the notion of $k$-determining are contained in Mather [1970], Siersma [1974] and Wasserman [1974], though they are not stated there in precisely the form we use here.

One of the goals of singularity theory is to bring functions into normal form in a neighborhood of a singular point. The procedure for doing so involves two steps:

1. The analytical step. This step gives criteria for when two functions are related by a diffeomorphism. This is done using what is called the deformation method and involves the integration of ordinary differential equations.

2. The algebraic step. The verification of the hypotheses needed to guarantee that a function is related to a specific normal form by a diffeomorphism usually reduces to a problem in linear algebra.

Let us formalize these steps somewhat, with a view toward the Morse lemma in $\mathbb{R}^{n}$. Let $g$ and $h$ be smooth real valued functions defined on a neighborhood of the origin in $\mathbb{R}^{n}$ with $g(0)=h(0)=0$. We say that $g$ and $h$ are right equivalent if there is a $C^{\infty}$ diffeomorphism $\phi$ defined on a neighborhood of 0 in $\mathbb{R}^{n}$ with $\phi(0)=0$ such that $g(x)=h(\phi(x))$. If $D \phi(0)=I=$ identity, we say that $g$ and $h$ are strongly right equivalent.

The Morse lemma in $\mathbb{R}^{n}$ states that if $g$ is a $C^{\infty}$ function satisfying $g(0)=0$ and $D g(0)=0$ and if $D^{2} g(0)$ is a nondegenerate symmetric bilinear form of index $k$ then $g$ is strongly right equivalent to

$$
h(x)=-\left(x_{1}^{2}+\cdots+x_{k}^{2}\right)+x_{k+1}^{2}+\cdots+x_{n}^{2} .
$$

The proof of the Morse lemma proceeds by two steps. First of all one shows that $g$ is strongly right equivalent to $Q(x)=\frac{1}{2} D^{2} g(0) \cdot(x, x)$ by writing $g=Q+p$ and seeking a diffeomorphism that eliminates $p$. The method for doing this is described below. Once this is done, linear algebra is used to make a further coordinate change to diagonalize $Q$. In this paper we shall concentrate on the first step; in infinite dimensions the second step depends on having a suitable spectral theorem available.

Now we discuss the general procedure one uses to show that $Q+p$ is right equivalent to $Q$. Let $\vartheta_{g}$ denote the set of functions which are right equivalent to $g$. Let $T g$ denote the formal tangent space to $\theta_{g}$ at $g$. More precisely, let $\phi_{t}$ be a curve of diffeomorphism with $\phi_{t}(0)=0$ and $\phi_{0}=\mathrm{Id}$; then $\psi_{t}(x)=g\left(\phi_{t}(x)\right)$ is a curve in $\theta_{g}$ with $\psi_{0}=g$. It follows from the chain rule that

$$
\left.\left(\frac{d}{d t} \psi_{t}\right)\right|_{t=0}(x)=D g(x) \cdot A(x)
$$

where $A(x)=\left.\dot{\phi}_{t}(x)\right|_{t=0}$. Thus, a typical element of $T g$ has the form $D g(x) \cdot A(x)$, where $A(0)=0$.

The deformation lemma is as follows:

Let $g_{t}=Q+t p$. Assume

(D1) $p \in T Q$,

(D2) $T g_{t}=T Q$ for all $t \in[0,1]$.

Then $Q+p$ is right equivalent to $Q$.

For strong right equivalence one modifies $T g$ to include the condition $D A(0)=0$, corresponding to $D \phi(0)=I$. A complete proof of the deformation lemma in the infinite dimensional case is given in the next section.

Next one uses the nondegeneracy hypotheses to show that indeed (D1) and (D2) are satisfied. For the Morse lemma this can be done directly since $D Q$ can be identified 
with an invertible linear transformation $T$ by $D Q(x) \cdot y=\langle x, T y\rangle$. Then in order to write $p(x)$ in the form $D Q(x) \cdot A(x)$, one can use Taylor's theorem to write $p(x)=$ $\langle x, \hat{p}(x)\rangle$ and let $A(x)=T^{-1} \hat{p}(x)$. This is how (D1) is checked. In fact, one has proved that if $p(x)$ vanishes at the origin, then $p$ is in $T Q$. Thus $T Q=\{p(x) \mid p(0)=0\}$.

One verifies $(D 2)$ by showing that $T g_{t}=\{p(x) \mid p(0)=0\}$ either by repeating the construction above for $\mathrm{Tg}_{t}$ or (preferably) by using a bit of algebraic machinery such as Nakayama's lemma. For more complicated singularities, the use of Nakayama's lemma is a practical necessity.

2. The deformation lemma. Let $E$ be a Banach space. Let $g, h: U \rightarrow \mathbb{R}$ be $C^{k}$ maps $(k \geq 1)$ defined on a neighborhood $U$ of $0 \in E$ and satisfy $g(0)=h(0)=0$. For $1 \leq l \leq k$, we shall say that $g$ is $C^{l}$ right equivalent to $h$ at 0 if there is a $C^{l}$ diffeomorphism $\phi$ : $V \rightarrow W$ of neighborhoods of 0 in $E$ such that

$$
\phi(0)=0 \text { and } g(x)=h(\phi(x)) \text { for all } x \in V .
$$

Furthermore, if $D \phi(0)=I$, the identity, we say that $g$ is $C^{l}$ strongly right equivalent to $h$ at 0 .

THEOREM A. Let $f$ and $p$ be $C^{k}$ real valued functions $(k \geq 1)$ defined on a neighborhood of 0 in $E$ and satisfy $f(0)=p(0)=0$. Make these assumptions:

(E1) There is an $l \geq 1$, and a $C^{l}$ map $A: U \rightarrow E$ defined on a neighborhood of 0 in $E$ such that

$$
A(0)=0 \text { and } p(y)=-D f(y) \cdot A(y) \quad \text { for } y \in U .
$$

(E2) There is a $C^{l}$ map $R: U \rightarrow L(E, E)$ (the bounded operators on $E$ with the norm topology) such that $R(0)=0$ and

$$
D p(y)=D f(y) \circ R(y) .
$$

Then $f+p$ is $C^{l}$ right equivalent to $f$ at 0 . Furthermore, if in $(E 1), D A(0)=0$, then $f+p$ is $C^{l}$ strongly right equivalent to $f$ at 0 .

Remarks. (a) Conditions (E1) and (E2) are precise versions of (D1) and (D2) in the previous section, with $Q$ replaced by $f$. Since $T f$ consists of functions of the form $D f(y) \cdot A(y)$, clearly $(E 1)$ is expressing the same ideas as $(D 1)$. For $(E 2)$, observe that

$$
D p(y)=D f(y) \circ R(y)
$$

is equivalent to saying that for all $t \in[0,1]$,

$$
D f(y)+t D p(y)=D f(y)+t D f(y) \circ R(y)
$$

i.e., with $f_{t}=f+t p$,

$$
D f_{t}(y)=D f(y) \circ L_{t}(y)
$$

where

$$
L_{t}(y)=I+t R(y)
$$

Thus there is a linear map relating $D f_{t}(y)$ and $D f(y)$. Since $R(0)=0, L_{t}(y)$ is invertible on a neighborhood of zero. Thus (E2) is expressing $(D 2)$. In fact the condition $R(0)=0$ can be replaced by $\|R(y)\|<1$ or invertibility of $L_{t}$ for the right equivalence conclusion.

(b) If $f$ is homogeneous of degree $\kappa$, i.e. $f(t y)=t^{\kappa} f(y)$ for a positive integer $\kappa$, and (E2) holds then $(E 1)$ holds. Also, if $R(0)=0$ then $D A(0)=0$ (so one has strong right 
equivalence). To see this use (E2) to write

$$
\begin{aligned}
p(y) & =\int_{0}^{1} D p(\tau y) \cdot y d \tau=\int_{0}^{1} D f(\tau y) \circ R(\tau y) \cdot y d \tau \\
& =\int_{0}^{1} \tau^{\kappa-1} D f(y) \circ R(\tau y) \cdot y d \tau .
\end{aligned}
$$

Thus, (E1) holds with $A(y)=-\int_{0}^{1} \tau^{\kappa-1} R(\tau y) \cdot y d \tau$. Note that $A(0)=0$ automatically and $R(0)=0$ implies $D A(0)=0$.

(c) Theorem A readily generalizes to the case in which $E$ is replaced by a Banach manifold. Condition ( $E 1)$ has intrinsic meaning independent of charts if $A$ is a vector field on $E$. Condition ( $E 2)$ also makes intrinsic sense if $R$ is a section of the bundle over $E$ whose fiber at $x \in E$ is the set of continuous linear maps of $T_{x} E$ to itself; this is a standard vector bundle associated with a manifold.

Proof of Theorem A. We first show that $f_{t}=f+t p$ is $C^{l}$ right equivalent to $f$ for small $t$. We find a curve of diffeomorphisms $\phi_{t}(x)=\phi(x, t)$ such that $\phi_{0}=I, \phi_{t}(0)=0$ and $f_{t}\left(\phi_{t}(x)\right)=f(x)$. To do this, we seek a vector field $A_{t}(x)=A(x, t)$ of class $C^{l}$ in $x$ and $t$ such that $A_{t}(0)=0$ and

$$
p(y)=-D f_{t}(y) \cdot A_{t}(y) .
$$

If $A_{t}$ is found, we let $\phi_{t}$ be its evolution operator defined by $\phi_{t}(x)=A_{t}\left(\phi_{t}(x)\right)$ and $\phi_{0}=I$. Then we have

$$
\frac{d}{d t} f_{t}\left(\phi_{t}(x)\right)=p\left(\phi_{t}(x)\right)+D f_{t}\left(\phi_{t}(x)\right) \cdot A_{t}\left(\phi_{t}(x)\right)=0 .
$$

Thus we get $f_{t}\left(\phi_{t}(x)\right)=f(x)$ as desired. Note that $A_{t}(0)$ implies $\phi_{t}(0)=0$ and $D A_{t}(0)=0$ implies $D \phi_{t}(0)=I$.

Note that $\phi_{t}$ can be defined (on some neighborhood of 0 ) for as long a $t$-interval as $A_{t}$ is defined. Indeed this follows from the fact that $A_{t}(0)=0$ and the continuous dependence of the solution curves on initial data. To construct $A_{t}$, we use (E2) to write

$$
D p(y)=D f(y) \circ R(y) \text {. }
$$

Therefore,

$$
D f_{t}(y)=D f(y)+t D p(y)=D f(y)(I+t R(y)) .
$$

Using this and $(E 1)$, the equation $D f_{t}(y) \cdot A_{t}(y)=-p(y)$ becomes

$$
D f(y)(I+t R(y)) \cdot A_{t}(y)=D f(y) \cdot A(y) .
$$

Since $R(0)=0, I+t R(y)$ is invertible for $y$ in a neighborhood of 0 and $0 \leq t \leq 1$, so we can take

$$
A_{t}(y)=(I+t R(y))^{-1} A(y) \text {. }
$$

3. Tromba's Morse lemma. This section shows that Tromba's Morse lemma is a direct and natural consequence of Theorem A. The setting is as follows.

Let $E$ be a Banach space. Let $\langle\cdot, \cdot\rangle$ be an inner product on $E$ and $B: E \times E \rightarrow \mathbb{R}$ a continuous symmetric bilinear form. Assume

$$
h: E \rightarrow \mathbb{R} \text { is } C^{k}, \quad k \geq 3,
$$


and satisfies:

$$
h(0)=0, \quad D h(0)=0, \quad D^{2} h(0)(u, v)=B(u, v) .
$$

Consider the following conditions:

(T1) There is a linear isomorphism $T: E \rightarrow E$ such that

$$
B(u, v)=\langle T u, v\rangle \text { for all } u, v \in E \text { (nondegeneracy). }
$$

(T2) $h$ has a $C^{k-1}$ gradient relative to $\langle\cdot, \cdot\rangle, \nabla h: U \rightarrow E$; i.e.,

$$
\langle\nabla h(y), u\rangle=D h(y) \cdot u \text {. }
$$

Note. (T1) implies that $T$ itself is symmetric (i.e. $\langle T u, v\rangle=\langle u, T v\rangle$ ), since $B$ is symmetric.

TheOREM B. (Morse-Tromba lemma). If $(T 1)$ and $(T 2)$ hold, then $h$ is $C^{k-2}$ strongly right equivalent to $f(x)=\frac{1}{2} B(x, x)$ at 0 .

Proof. Note that $D f(y) \cdot u=B(y, u)=\langle T y, u\rangle$, so $\nabla f(y)=T y$. By Taylor's theorem, write $h=f+p$ where $p$ is $C^{k}$, has a gradient and $p(0)=0, D p(0)=0$ and $D^{2} p(0)=0$.

Since $f$ is quadratic, Remark (b) following Theorem $\mathrm{A}$, shows that it suffices to show that ( $E 2)$ holds with $R(0)=0$.

To do this, use the fact that $p$ has a $\langle\cdot, \cdot\rangle$ gradient to write

$$
\begin{aligned}
D p(y) \cdot u & =\langle\nabla p(y), u\rangle \\
& =\left\langle\int_{0}^{1} D \nabla p(\tau y) \cdot y d \tau, u\right\rangle .
\end{aligned}
$$

However, differentiating $D p(x) \cdot u=\langle\nabla p(x), u\rangle$ in $x$, we see that $D \nabla p(x)$ is symmetric. Thus

$$
D p(y) \cdot u=\left\langle y,\left(\int_{0}^{1} D \nabla p(\tau y) \cdot y d \tau\right) \cdot u\right\rangle .
$$

Hence we can take $R(y)=\int_{0}^{1} D \nabla p(\tau y) \cdot y d \tau$.

Note that $R(0)=0$ so we have strong right equivalence, as required.

Remarks. (a) There are versions of this theorem for which $E$ is a Banach manifold. The main difference is to let $\langle\cdot, \cdot\rangle$ depend on the base point. These versions can also be derived from Theorem A. Such generalizations are called for in minimal surfaces (see Tromba [1981]) and in fluid mechanics where $E$ is a coadjoint orbit (see Arnold [1978, App. 5] and Ebin and Marsden [1970]).

(b) If the hypothesis $(T 1)$ of Theorem $\mathrm{B}$ holds and if $R(y)$ exists and has a $C^{k-2}$ adjoint $R(y)$ i.e.

$$
\left\langle R(y)^{*} u, v\right\rangle=\langle u, R(y) v\rangle,
$$

then (T2) holds.

Indeed, $D f(y) \circ R(y)=D p(y)$, so

$$
\langle T y, R(y) \cdot u\rangle=D p(y) \cdot u,
$$

so

$$
\nabla p(y)=R(y)^{*} \cdot T y .
$$


Thus, if $(T 1)$ and (E2) hold, the extra condition that Theorem $\mathrm{B}$ requires is the existence of $R(y)^{*} \cdot T y$. The $R(y)$ chosen in the proof of Theorem B is symmetric, but even when (T1) holds it is possible to satisfy Theorem A even when $R(y)^{*} \cdot T y$ does not exist. An example is given in the following paper.

(c) If (T1) holds for two different inner products $\langle\cdot, \cdot\rangle_{1}$ and $\langle\cdot, \cdot\rangle_{2}$, then $(T 2)$ will hold for $\langle\cdot, \cdot\rangle_{1}$ if and only if it does for $\langle\cdot, \cdot\rangle_{2}$. Indeed, using obvious notation, the two gradients are related by

$$
\nabla_{1} h(y)=T_{2} T_{1}^{-1} \nabla_{1} h(y) .
$$

Thus, changing the weak metric does not aid in the existence of the gradient. Likewise, allowing $\langle\cdot, \cdot\rangle$ to depend on the base point, but assuming $\langle u, v\rangle_{x}=\left\langle T_{x} u, v\right\rangle_{0}$ for an isomorphism $T_{x}$ (perhaps chart dependent) does not help with the existence of the gradient.

In fact, $\langle\cdot, \cdot\rangle$ can be any continuous symmetric bilinear form, degenerate or not and the proof still goes through. One can always choose $\langle\cdot, \cdot\rangle=B(\cdot, \cdot)$ although it may be computationally convenient to make a different choice. However, for the splitting lemma considered below, where $T$ need not be invertible, the choice of $\langle\cdot, \cdot\rangle$ really will affect whether or not the gradient exists.

(d) Assume (T1) holds for $f(x)=\frac{1}{2} B(x, x)$ and let $p(x)=h(x)-f(x)$, where $h$ satisfies the conditions preceeding (T1). Then conditions $(E 1)$ and $(E 2)$ hold if and only if there exists a $C^{l}$ map $\tilde{R}: U \rightarrow L(E, E)$ defined on a neighborhood $U$ of 0 in $E$ such that $\tilde{R}(0)=0$ and

$$
D p(y) \cdot u=\langle y, \tilde{R}(y) \cdot u\rangle \quad \text { for all } y \in U \text { and } u \in E .
$$

Indeed, if $(R)$ holds, $(E 1)$ and $(E 2)$ are verified with $A(y)=-T^{-1} \int_{0}^{1} \tilde{R}(\tau y) \cdot \tau y d \tau$ and $R(y)=T^{-1} \tilde{R}(y)$. Conversely (E2) gives condition $(R)$ with $\tilde{R}(y)=T R(y)$.

(e) If $E$ is finite dimensional or admits a " $C^{k}$ duality mapping", then Theorem B can be improved to $k \geq 2$ and the right equivalence is $C^{k-1}$ (and $C^{k}$ away from 0 ). In finite dimensions this result is due to Kuiper [1972] and in infinite dimensions to Tuan and Ang [1979]. The same result can be proved by the methods of this paper by using the Whitney properties of the remainder term in the form given by Tuan and Ang [1979].

4. The splitting lemma. We now briefly discuss the splitting lemma of Gromoll and Meyer [1969], which enables one to reduce infinite dimensional catastrophe theory to the finite dimensional case. As usual, we want hypotheses that will be applicable to elliptic variational problems - see the following paper for specific examples. We shall work in the context of Theorem B.

Let $E$ be a Banach space and $h: E \rightarrow \mathbb{R}$ be $C^{k}, k \geq 3$ defined in a neighborhood $U$ of 0 . Suppose $h(0)=0$ and write $D^{2} h(0)(u, v)=B(u, v)$. Let $\langle\cdot, \cdot\rangle$ be an inner product on $E$ and assume

(S1) there is a Fredholm operator $T: E \rightarrow E$ of index 0 such that $B(u, v)=\langle T u, v\rangle$ for all $u, v \in E$.

Since $T$ is Fredholm of index 0 , we can write $E=K \oplus L$, where $K=\operatorname{ker} T$ and $L=$ range $T$ (note that $T$ is symmetric). Denote points in $K \oplus L$ as pairs $(x, y)$. Also assume:

$(S 2)=(T 2) h$ has a $C^{k-1}$ partial gradient $\nabla_{y} h: U \rightarrow L$ (i.e. $\left\langle\nabla_{y} h(u), v\right\rangle=D h(u) v$ for all $v \in L)$ and $\nabla_{y} h(0,0)=0$. 
SPLITTING LEMMA. There is a change of coordinates $\bar{x}=x, \bar{y}=\eta_{x}(y)=\eta(x, y)$ such that in a neighborhood of $(0,0), h$ has the form

$$
h(\bar{x}, \bar{y})=\frac{1}{2} D_{y}^{2} g(0,0)(\bar{y}, \bar{y})+r(\bar{x}),
$$

where $\eta(0,0)=0, D_{x} \eta(0,0)=0, D_{y} \eta(0,0)=I$ and $r(0)=0, D r(0)=0$ and $D^{2} r(0)=0$.

Proof. Consider

$$
\nabla_{y} h: U \rightarrow L .
$$

Let $P$ be the projection of $E \rightarrow L$ whose kernel is $K$, and note that $P \circ T$ restricted to $L$ is an isomorphism of $L$ to itself. Observe that $D_{y} \nabla_{y} h(0,0): L \rightarrow L$ is the operator $P \circ T$. Thus, the implicit function theorem guarantees that the equation

$$
\nabla_{y} h(x, y)=0, \text { i.e., } \quad D_{y} h(x, y)=0
$$

uniquely defines a function $y=F(x)$ near $(0,0) ; F(0)=0$ and $F^{\prime}(0)=0$.

Let $g(x, y)=h(x, y+F(x))$ and note that

$$
D g(x, y)=D h(x, y+F(x)) \circ\left(\begin{array}{cc}
I & 0 \\
F^{\prime}(x) & I
\end{array}\right) \text {. }
$$

An easy computation shows that $D g(x, y)=0$ implies $y=0$ and $D_{x} g(x, 0)=0$ if and only if $\operatorname{Dh}(x, F(x))=0$.

Theorem B (with $h$ depending on parameters) shows that there exists an $x$-dependent change of coordinates $y=\Phi_{x}(y)$ such that

$$
g(x, \hat{y})=\frac{1}{2} D_{y}^{2} g(x, 0)(\hat{y}, \hat{y})+g(x, 0) .
$$

Theorem A can now be applied in a similar way as in the proof of Theorem B to the first term $\frac{1}{2} D_{y}^{2} g(x, 0)(\hat{y}, \hat{y})$ showing that there is a further coordinate change $\tilde{y}=\Psi_{x}(\hat{y})$ depending parametrically on $x$, which transforms this term to $\frac{1}{2} D_{y}^{2} g(0,0)(\tilde{y}, \tilde{y})$. Putting these coordinate changes together gives the result.

Remarks. 1. Letting $m=\operatorname{dim} K$, this splitting lemma is a generalization of the usual form of the splitting lemma: a function whose Hessian has corank $m$ at a critical point can be decomposed as the sum of a 2-flat function of $m$-variables and a nondegenerate quadratic form in the remaining variables. See, for example, Wasserman [1974, p. 137].

2. The proofs we give are for $k \geq 3$ and give coordinate changes of class $k-2$. However, as in remark (e) following Theorem B, this can be improved to $k \geq 2$ and coordinate changes of class $C^{k-1}$ if the Banach space $L$ admits a $C^{k}$ duality mapping.

3. To find the critical points of $h$, it is enough to find the critical points of $g$ restricted to the finite dimensional space $K=\operatorname{ker} T$.

4. To find a normal form for $h$, it is enough to find one for $g \mid K$. Note that the computation of $g$ is not necessarily an easy matter as its definition depends on the implicitly defined function $F$. However, using implicit differentiation one can in principle calculate the Taylor expression of $g$ to any given order.

Since $K$ is finite dimensional, ordinary catastrophe theory can be used to classify generically what happens in these situations. If $h$ depends on parameters, the splitting lemma is to be used in a parametric way; a specific example is worked out in the following paper (see Example 7). The general results of Chillingworth [1980] also generalize to the present context. Finally, we refer to Magnus [1980] for a splitting lemma under hypotheses similar to those described here. 


\section{REFERENCES}

L. ARKERYD [1979], Thom's theorem for Banach spaces, J. London Math. Soc., 19, pp. 359-370.

J. ARMS, J. MARSDEN AND V. MONCRIEF [1982], The structure of the space of solutions of Einstein's equatios II: Several Killing fields and the Einstein-Yang-Mills equations, Ann. Phys., 144, pp. 81-106.

V. ARNOLd [1978], Mathematical Methods of Classical Mechanics, Springer, New York.

J. M. BALl, R.J, KNOPS AND J. E. MARSDEN [1978], Two examples in nonlinear elasticity, Lecture Notes in Mathematics 166, Springer, New York, pp. 41-49.

M. BeESON AND A. J. TROMBA [1981], The cusp catastrophe of Thom in the bifurcation of minimal surfaces, preprint \#462, SFB, Bonn, Manus. Math. to appear.

M. BUCHNER, J. MARSDEN AND S. SCHECTER [1983], Examples for the infinite dimensional Morse lemma, this Journal, this issue, pp. 1045-1055.

D. Chillingworth [1980], A global genericity theorem for bifurcations in variational problems, J. Funct. Anal., 35, pp. 251-278.

Y. Choquet-Bruhat AND J. MARSDen [1976], Solution of the local mass problem in general relativity, Comm. Math. Phys. 51, pp. 283-296.

G. DANGelmaYR [1979], Catastrophes and bifurcations in variational problems, in Structural Stability in Physics, Guttinger and Elkemeier, eds., Springer-Verlag, New York.

D. EBIN AND J. MARSDEN [1970], Groups of diffeomorphism and the motion of an incompressible fluid, Ann. Math., 92, pp. 102-163.

D. Gromoll AND W. MeYer [1969], On differentiable functions with isolated critical points, Topology, 8, pp. 361-369.

N. H. KUIPER [1972], $C^{1}$-equivalence of functions near isolated critical points, in Symposium on Infinite-Dimensional Topology, Annals of Math. Studies 69, R. D. Anderson, ed., Princeton Univ. Press, Princeton, NJ

R. J. Magnus [1976], On universal unfoldings of certain real functions on a Banach space, Math. Proc. Camb. Phil. Soc., 81, pp. 91-95.

[1978], Determining in a class of germs on a reflexive Banach space, Proc. Camb. Phil. Soc., 84, pp. 293-302.

[1979], Universal unfoldings in Banach spaces: reduction and stability, Math. Proc. Camb. Phil. Soc., 86, pp. 41-55.

[1980], A splitting lemma for nonreflexive Banach spaces, Math. Scand. 46, pp. 118-128.

J. Marsden And T. Hughes [1983], Mathematical Foundations of Elasticity, Prentice-Hall, Englewood Cliffs, NJ

J. MATHER [1970], Notes on right equivalence, Univ. of Warwick, preprint.

R. PALAIS [1963], Morse theory on Hilbert manifolds, Topology 2, pp. 299-340. [1969], The Morse lemma on Banach spaces, Bull. Amer. Math. Soc. 75, pp. 968-971.

D. SiERSMA [1974], Classification and deformation of singularities, Thesis, Amsterdam.

A. J. Tromba [1976], Almost-Riemannian structures on Banach manifolds, the Morse lemma and the Darboux theorem, Canad. J. Math., 28, pp. 640-652.

A. J. Tromba [1981], A sufficient condition for a critical point of a functional to be a minimum and its application to Plateau's problem, preprint \#408 SFB, Bonn. Math. Ann. (to appear).

V. T. Tuan AND D. D. ANG [1979], A representation theorem for differentiable functions, Proc. Amer. Math. Soc., 75, pp. 343-350.

G. WASSERMAN [1974], Stability of Unfoldings, Lecture Notes in Mathematics 393, Springer, New York. 